\title{
Data dissemination with rateless coding in a grid vehicular topology
}

\author{
Mostafa Asgharpoor Salkuyeh ${ }^{1}$, Faramarz Hendessi ${ }^{1}$ and T Aaron Gulliver ${ }^{2^{*}}$
}

\begin{abstract}
Vehicular ad hoc networks are an important new class of wireless networks with applications ranging from safety and crash avoidance to Internet access and multimedia distribution. In this article, an efficient method of message dissemination using rateless coding is proposed. Both vehicle-to-vehicle and vehicle-to-infrastructure data transfer are allowed. By employing rateless coding at road side units and using vehicles as data carriers, messages can be propagated efficiently. To limit the buffer capacity required in vehicles, buffer management is employed. The network considered has a grid topology. The effects of vehicle speed, number of lanes, broadcast interval, and decoding distance on the performance are examined.
\end{abstract}

Keywords: Ad hoc network, Data dissemination, Rateless coding, RSU, VANET

\section{Introduction}

The need of humans to be connected has led to rapid growth in wireless communications technology. Vehicular ad hoc networks (VANETs) are an emerging part of this trend. These networks can be used to disseminate information such as weather, traffic conditions, and commercial information such as restaurant and gas station locations. The many VANET applications can be divided into two main categories: safety and non-safety. Safety applications include spreading an alarm or warning with the aim of avoiding danger and reducing risk. Non-safety applications include information about a new product or business, the closest restaurant or gas station, or the shortest path to a destination. This information may be transmitted in response to a request. Since this information may include multimedia content, the message size can be considerably greater than that of safety messages. Road side units (RSUs) must therefore distribute messages of varying sizes and content, and this is done according to time, current policy, traffic conditions, etc.

The applications mentioned above are just the beginning of the flood of applications anticipated for VANETs. As with other types of networks, VANETs have their own

\footnotetext{
* Correspondence: agullive@ece.uvic.ca

${ }^{2}$ Department of Electrical and Computer Engineering, University of Victoria, P.O. Box 3055, STN CSC, Victoria, BC V8W 3P6, Canada

Full list of author information is available at the end of the article
}

advantages and disadvantages. VANETs are a subset of mobile ad hoc networks (MANETs) with restricted node behavior. Vehicle (node) velocity and their limited ability to change direction are two significant differences between MANETs and VANETs. Due to their highly dynamic and restricted characteristics, VANETs are more vulnerable to the fragmentation problem. This means that delivery of a message to an arbitrary vehicle can be very difficult. One of the proposed methods for mitigating this problem is store-carry-forward (SCF) [1].

Every RSU broadcasts messages based on time, policy, or network-specific criteria. Vehicles approaching an RSU receive these messages, and they should be decoded and a decision made before passing the RSU. For example, suppose a multimedia message which contains a gas station location is being transmitted. A vehicle looking for a gas station should decode this message before the corresponding RSU is encountered. In other words, the vehicle must be given sufficient time to make a decision, safely reduce speed, and possibly change direction. Every message has a validation period, after which it is replaced with another message according to the corresponding policy.

The distance between a vehicle with a message and the source of that message is called the decoding distance. In this article, the decoding distance is increased by combining rateless coding with the SCF technique. The tradeoffs between vehicle speed, broadcast interval, 
number of lanes, and decoding distance are examined. Due to buffer limitations, we employ a buffer management mechanism. The impact of this mechanism is investigated. The effect of an RSU changing its messages, and the ability of vehicles to sense these changes, is also examined. Finally, message dissemination patterns around each RSU are determined. This shows the effectiveness of the proposed approach.

The remainder of this article is organized as follows. Section 2 provides a review of related work, and Section 3 gives a brief introduction to rateless coding. Data dissemination in VANETs is introduced in Section 4. The network model and the assumptions used in the analysis are given in Section 5. Performance results are presented and explained in Section 6. Finally, some conclusions are given in Section 7.

\section{Related work}

Data dissemination in VANETs has been the subject of significant research in recent years. Various dissemination techniques, particularly for safety applications, have been developed. Techniques have also been proposed to provide multimedia data, social networking, and traffic information.

Initial studies on data dissemination to multiple nodes have been done in $[2,3]$. The problem of data dissemination from an RSU to vehicles on a highway is considered in [4]. In [5], network coding was employed for video streaming in VANETs. However, broadcasting this type of data can lead to severe congestion and significantly reduce the data delivery ratio. Zhao et al. [6] proposed the data pouring (DP) method to help solve this problem. DP exploits the predictability of vehicle mobility due to the road configuration. However, they assumed only one data source, and unlimited buffers in vehicles for storing information.

The cooperative rateless code protocol (CORP) was proposed in [7]. Data dissemination with CORP has two phases. In the first phase, vehicles receive rateless coded information from access points which can be fixed infrastructure or mobile nodes. In the second phase, information is disseminated through the network via vehicle cooperation. In this phase, vehicles that have received a sufficient number of encoded symbols (ESs) can generate new ESs and disseminate them in the network. Such a vehicle is called a certified car. CORP uses a complex six-way handshaking protocol with limited cooperation, which is not well suited to short-lived connections. In addition, only freeway vehicle traffic was considered, along with unlimited capacity vehicle buffers, which is unrealistic. In [8], fountain codes were employed for data dissemination in VANETs. The Vimesh protocol was presented which can provide covert operation via directional antennas. However, the resulting performance can be poor due to the unreliability of time-varying communications channels. Note that the road topology was not considered in $[7,8]$, even though this is the major distinguishing feature between vehicular networks and MANETs. Limitations on vehicle movement can have a significant effect on vehicle cooperation, and thus must be considered in evaluating VANET communications systems.

Employing rateless coding for data dissemination has also been investigated in $[9,10]$ for bidirectional single lane roads. In [11], rateless coding was employed with SCF in an urban topology. However, only the performance at the junction of two roads was investigated. In this article, these techniques are considered in a grid topology with multiple intersections.

A new data dissemination technique for urban vehicular networks is presented here. Unlike with CORP, vehicles retransmit packets as opposed to ESs, and there is no restriction on which packets can be transmitted. This reduces system complexity and overhead. Finite vehicle buffers are also considered as a practical limitation, and a buffer management scheme is presented.

\section{Rateless codes}

Traditional techniques for data transfer over erasure channels require continuous two-way communications. Packets are encoded and transmitted to the receiver which then attempts to decode them. If decoding is successful, an acknowledgment is transmitted to the sender. Otherwise, the receiver requests retransmission of the erroneous packets. However, systems such as broadcast networks may not have a feedback channel for acknowledgements. Rateless coding provides a means of solving this problem by allowing a one-way communication protocol. In this case, packets are still encoded and sent to the receiver. If a packet is received in error, it is simply discarded. Otherwise, the packet is retained as part of the message. The receiver is able to reconstruct the message after collecting a sufficient number of valid packets.

Rateless coding is an efficient way to combat channel uncertainty. The code rate automatically adapts to the channel quality through the use of incremental redundancy. Unlike conventional coding schemes, a rateless encoder can generate codewords with arbitrary length [12]. Therefore, very long codewords can be used as needed rather than employing a fixed rate code [13]. This is an effective solution for time-varying communications channels.

A key advantage of rateless codes is the existence of low complexity encoding and decoding algorithms [2]. It is shown in [5] that the receiver needs at least $k \Gamma_{\mathrm{k}}$ distinct packets to be able to decode $k$ data packets with probability $1-\delta . \Gamma_{\mathrm{k}}$ is the decoding overhead and is given by 


$$
\Gamma_{k}=1+O\left(k \log \frac{k}{\delta}\right)
$$

To encode a message, a random number $z$ is first generated as in [5]. Then, $z$ packets are randomly selected based on a uniform probability density function (PDF). Next, these $z$ packets are added modulo 2 (XOR) to obtain a new packet. The indexes of the encoded packets are added to the generated packet. This process can be repeated as many times as necessary to obtain a codeword of arbitrary length. In time-varying channels such as wireless fading channels, receivers may need many more packets, thank the minimum to successfully recover a message. The number of packets required for successful decoding depends greatly on the network and channel characteristics. Figure 1 depicts packet generation for a rateless code. The data packets are $\mathrm{P} 1, \mathrm{P} 2, \ldots$, $\mathrm{P} n$ with indexes ID1, ID2,...,IDn, respectively. The XOR of the $z$ selected packets results in a packet Pnew. The indexes of the $z$ selected packets are added to this new packet as IDnew.

\section{Data dissemination}

In this section, the method proposed in [10] for data dissemination in VANETs is reviewed. The fundamental network parameters are examined, and the simulation environment explained.

\subsection{Data Dissemination with Rateless Coding}

In order to disseminate a message, an RSU divides it into packets suitable for encoding using a rateless encoder. Once this process is completed, the resulting packets are disseminated in the network by the RSU.

When a vehicle collects a sufficient number of packets, it will be able to decode the message. Then the message is again divided into packets and encoded. Thus, a vehicle which successfully receives a message becomes a carrier for that message. It acts as a mobile representative for the RSU because it helps disseminate the message while traveling through the network.
A vehicle can be a carrier for some RSUs while being just a collector for others. A vehicle is free to decode any messages received while moving in the network. Therefore, it can carry message packets from multiple RSUs. However, vehicles have limited buffer capacity, and consequently they may not be able to save all received messages. When a vehicle with a full buffer encounters a new message, it discards the packets of the oldest message to create room for the new message. Since RSUs may disseminate messages which change over time, vehicles must update the messages being carried. This process consists of discarding old message packets and collecting the corresponding new ones.

In this article, it is assumed that every vehicle has sufficient capacity to store five messages. Thus, a buffer is divided into five message spaces. A maximum number of packets is assumed for each message, and this determines the buffer capacity. Every RSU appends its own ID and a message ID to the header of each packet to be disseminated. When a vehicle receives a packet from an RSU, it first extracts the RSU and message IDs from the header. If packets from this RSU are in the vehicle buffer, the vehicle checks the message ID. If the ID from the newly received packet is equal to the ID of the message in the buffer, the packet is added to the buffer. If the new packet ID differs from the ID of the message in the buffer, a message change has occurred. In this case, the packets from the old message are discarded and the newly received packet is stored in the buffer. If no packets from this RSU exist in the buffer, there are two possibilities:

1. There is a free message space in the vehicles buffer. In this case, the newly received packet is stored there.

2. There is no free message space in the buffer. In this case, the oldest message is discarded and the newly received packet is stored in the corresponding space.

In both cases, the time the packet was received is also stored so that the age of the message can be determined.

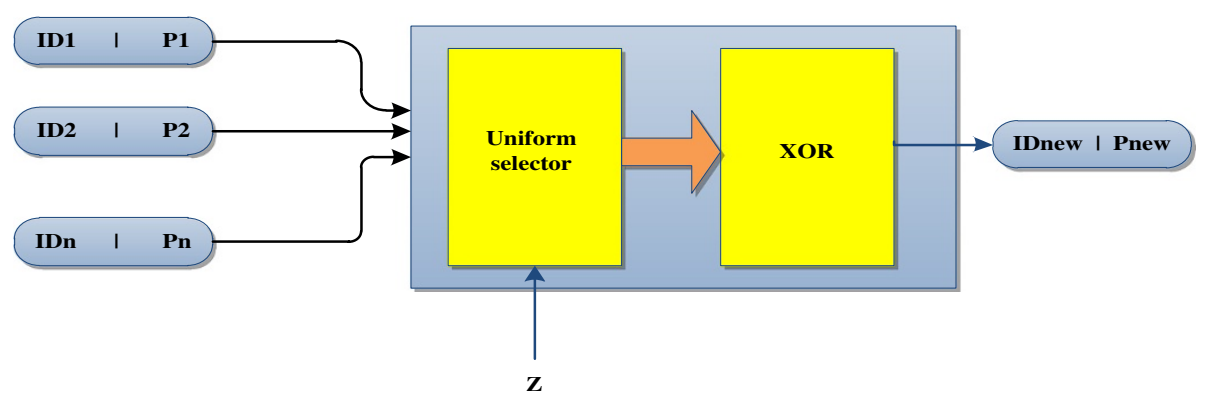

Figure $1 \mathrm{~A}$ rateless code encoder. 
After a vehicle collects enough packets to be able to decode a message, it is decoded and then re-encoded. These newly created packets are then broadcast by the vehicle. Such a vehicle is called a carrier. To improve the dissemination process, vehicles broadcast all packets in their buffer while receiving packets from RSUs and other vehicles. Thus, every vehicle is involved in the dissemination process.

\section{Network model}

In this section, a model of the vehicle mobility is developed and the network-specific parameters are derived. We first consider sparse network topologies. In [14], it was shown that the inter-vehicle spacing can be considered to have an exponential distribution with parameter $\lambda_{s}$

$$
f_{S}(s)=\lambda_{s} e^{-\lambda_{s} s} .
$$

This is used below to determine the system parameters.

\subsection{Mobility model}

Define a cluster as a group of vehicles which are close together while moving such that they can communicate with each other. We first consider the number of clusters encountered by a vehicle while traveling a distance of $L$ (in meters) on a road. The expected number of clusters encountered is given by

$$
E\left[M_{n}(L)\right]=\frac{L}{E\left[c_{\text {length }}\right]+E\left[s_{\text {inter }}\right]}+E\left[r_{L}\right]+E\left[N_{\text {clus inter }}\right],
$$

where $E\left[c_{\text {length }}\right]$ is the expected length of a cluster in meters, $E\left[s_{\text {inter }}\right]$ is the expected distance between two successive clusters, $\left[E\left[r_{L}\right]\right.$ is the expected number of new vehicles that enter the road during the corresponding time interval and $E\left[N_{\text {clus inter }}\right]$ is the average number of clusters encountered in an intersection.

The distribution of the inter-vehicle spacing is required to determine $E\left[s_{\text {inter }}\right]$ and is given by

$$
\begin{aligned}
f_{S_{\text {inter }}}\left(s_{\text {inter }}\right) & =p_{r}\left(s_{\text {inter }} \mid s_{\text {inter }}>R\right)=\frac{\lambda_{s} e^{-\lambda_{s} s_{\text {inter }}}}{e^{-\lambda_{s} R}}, \\
& =\lambda_{s} e^{-\lambda_{S}\left(s_{\text {inter }}-R\right)}
\end{aligned}
$$

where $R$ is the communication range of a vehicle in meters. Using (4), we have

$$
E\left[s_{\text {inter }}\right]=\int_{R}^{\infty} s_{\text {inter }} \times \lambda_{s} e^{-\lambda_{s}\left(s_{\text {inter }}-R\right)} d s_{\text {inter }}=R+\frac{1}{\lambda_{s}} .
$$

Figure 2 shows $E\left[s_{\text {inter }}\right]$ with respect to $\lambda_{s}$.

To determine $E\left[c_{\text {length }}\right]$, the average length of a cluster, we first calculate the average number of vehicles in each cluster. Let $s_{i}$ be the distance between vehicles $i$ and $i+1$. The probability of having $n$ vehicles in a cluster, denoted $f_{c}(n)$, is given by

$$
\begin{aligned}
& f_{c}(1)=p\left(s_{1}>R\right)=p_{d} \\
& f_{c}(2)=p_{r}\left(s_{1} \leq R, s_{2} \geq R\right)=\left(1-p_{d}\right) p_{d} \\
& \vdots \\
& f_{c}(n)=\left(1-p_{d}\right)^{n-1} p_{d}
\end{aligned}
$$

and thus the expected number of vehicles in a cluster is

$$
E[c]=\frac{1}{p_{d}}
$$

where $p_{d}=p(s>R)=e^{-\lambda_{s} R}$ is the probability that the distance between two successive vehicles is more than

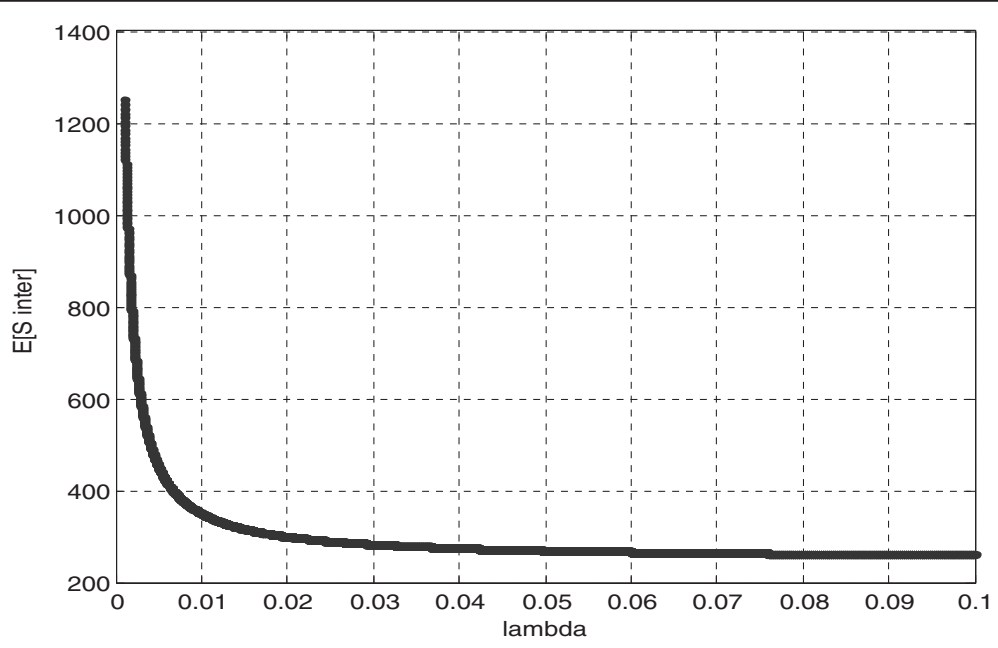

Figure $2 E\left[s_{\text {inter }}\right]$ versus $\lambda_{s}$. 


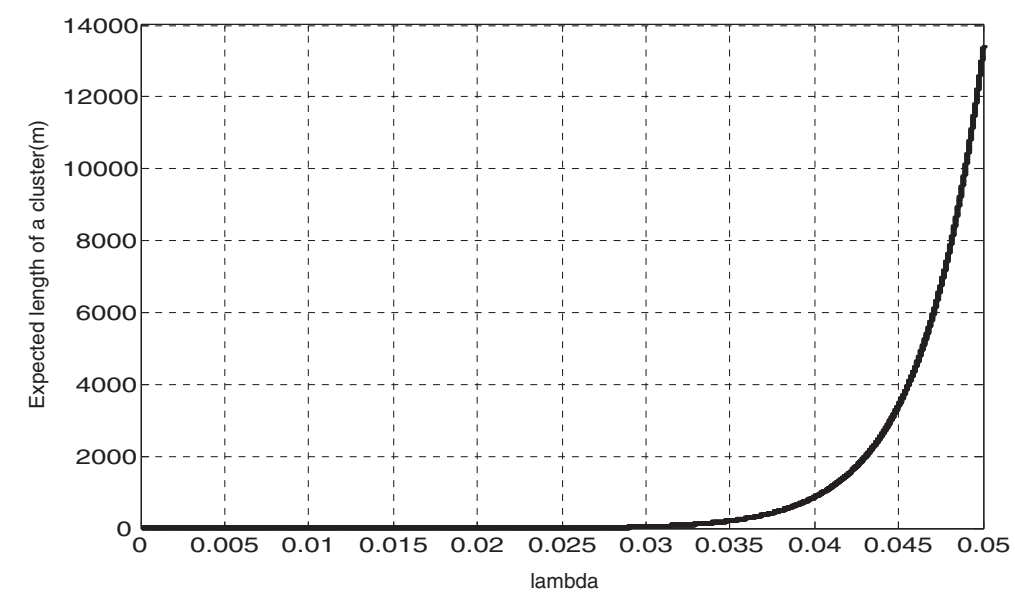

Figure $3 E\left[c_{\text {length }}\right]$ versus $\lambda_{\text {s. }}$

the communication range $R$. Figure 3 shows $E\left[c_{\text {length }}\right]$ with respect to $\lambda_{s}$.

The PDF of the vehicle spacing in a cluster is

$$
f_{S_{\text {intra }}}\left(s^{*}{ }_{\text {intra }}\right)=p\left(s_{\text {intra }} \mid s_{\text {intra }} \leq R\right)=\frac{\lambda_{s} e^{-\lambda_{s} s^{*} \text { intra }}}{1-e^{-\lambda_{s} R}}
$$

and therefore

$$
E\left[s_{\text {intra }}\right]=\frac{1}{\lambda_{s}}-\frac{e^{-\lambda_{s} R}}{1-e^{-\lambda_{s} R}} .
$$

Consequently, $E\left[c_{\text {length }}\right]$ can be expressed as

$$
E\left[c_{\text {length }}\right]=E[c] \times E\left[s_{\text {intra }}\right]=\frac{1}{p_{d}}\left(\frac{1}{\lambda_{s}}-\frac{e^{-\lambda_{s} R}}{1-e^{-\lambda_{s} R}}\right) .
$$

Let $\lambda_{t}(\mathrm{veh} / \mathrm{s})$ be the vehicle arrival rate with $\lambda_{t}=\lambda_{s} \times v$, where $v$ is the vehicle velocity. Define $t_{L}$ as the time it takes in seconds for a vehicle to travel $L$ meters along a road. The average number of new vehicles that enter the road during this time period is then $\lambda_{t} t_{L}$. To determine $t_{L}$, we first consider the vehicle speed, which is assumed to be given by

$$
v\left(\lambda_{s}\right)=v_{\max }\left(1-\frac{\lambda}{\lambda_{\max }}\right),
$$

where $v_{\max }$ is the maximum allowable speed and $\lambda_{\max }$ is the maximum capacity of the road. Figure 4 shows the relationship between time and vehicle speed for this road length. In the first half of the time interval, the vehicle accelerates up to a maximum, and in the second half it de-accelerates to a full stop.

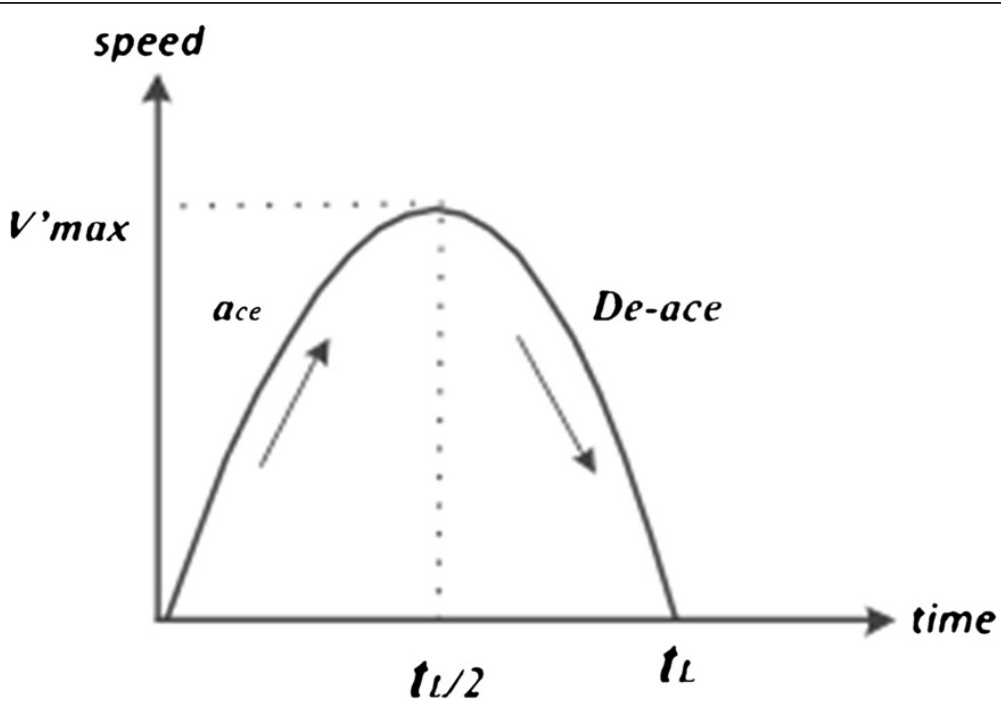

Figure 4 Vehicle speed versus time. 
Let $x$ be the vehicle position. Given the above assumptions, the position in the first half of the road is given by

$$
x=\frac{t^{2}}{2} a_{c c} \text { so that } \frac{L}{2}=\frac{1}{2} a_{c c}\left(\frac{t_{L}}{2}\right)^{2},
$$

and therefore the acceleration is given by

$$
a_{c c}=L / t_{L / 2}^{2} \text {. }
$$

The maximum speed for a vehicle with respect to the current traffic conditions can be obtained from (8). Let this speed be $v_{\max }^{\prime}$. Then $a_{c c}=\frac{\Delta v}{\Delta t}=\frac{v_{\max }^{\prime}}{t_{L / 2}}$, which from (10) gives $t_{L / 2}=\frac{L}{v_{\max }}$ so that

$$
t_{L}=\frac{2 L}{v_{\max }^{\prime}} .
$$

Therefore, we have

$$
\begin{aligned}
E\left[M_{n}(L)\right]= & \frac{L}{E\left[c_{\text {length }}\right]+E\left[s_{\text {inter }}\right]}+\lambda_{s} \frac{2 L}{v_{\max }^{\prime}}+E\left[N_{\text {clus inter }}\right] \\
= & \frac{L}{R+\frac{1}{\lambda_{s}}+\frac{1}{p_{d}}\left(\frac{1}{\lambda_{s}}-\frac{e^{-\lambda_{s} R}}{1-e^{-\lambda_{s} R}}\right)} \\
& +\lambda_{s} \frac{2 L}{v_{\max }^{\prime}}+E\left[N_{\text {clus inter }}\right]
\end{aligned}
$$

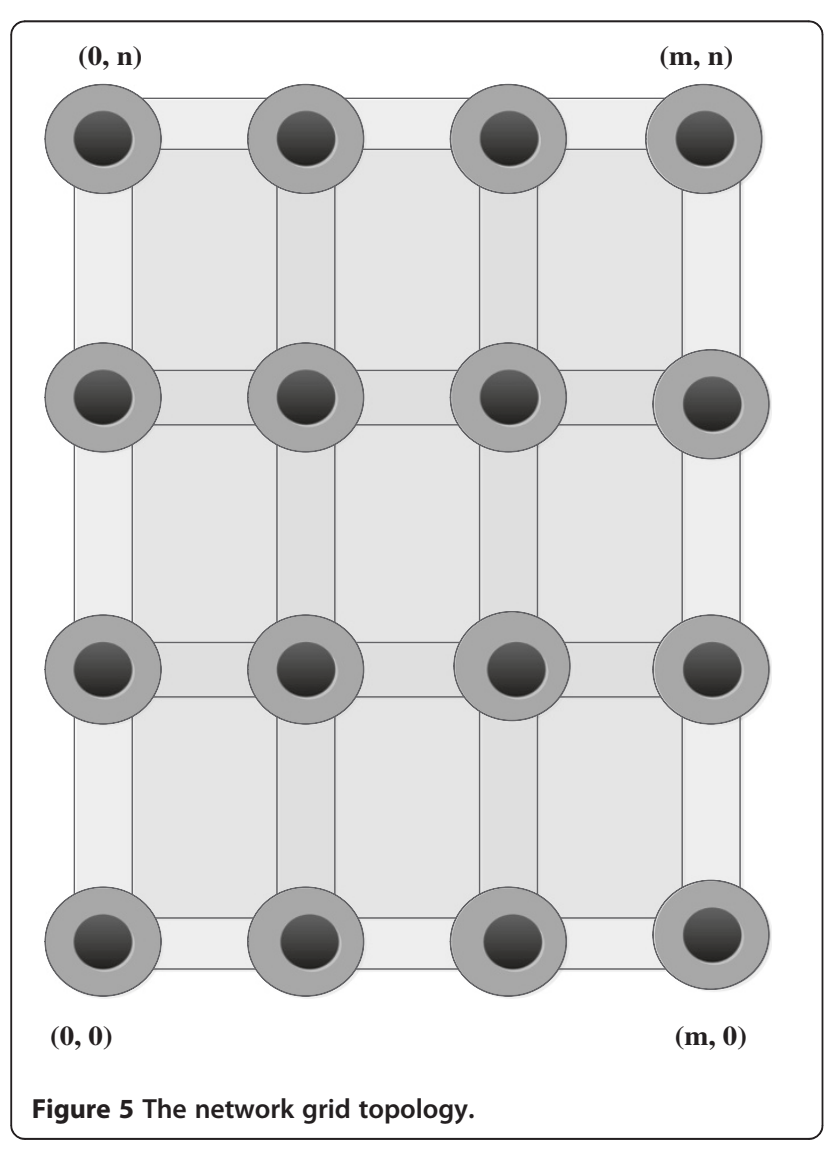

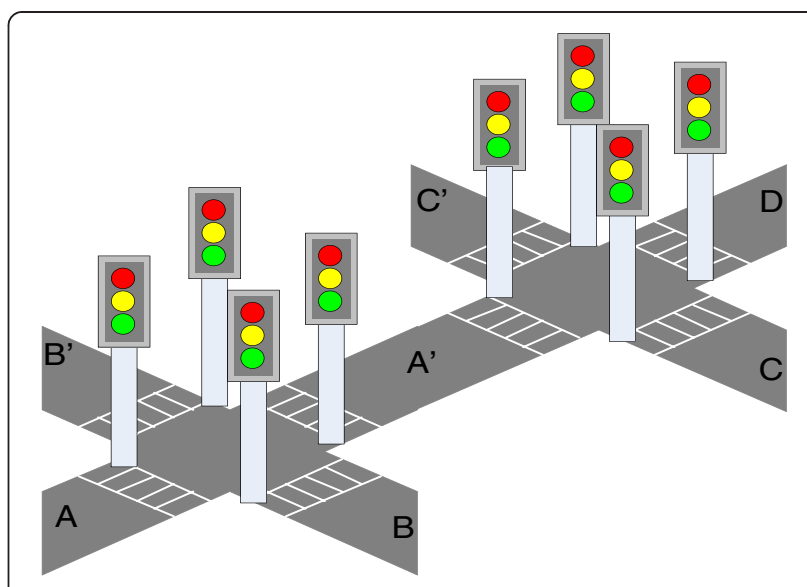

Figure 6 Two connected intersections equipped with traffic lights.

Since roads are not all the same length, we require the average value of $t_{L}$. This is given by

$$
\begin{aligned}
E\left[t_{L}\right] & =2 E\left[\frac{L_{\text {road }}}{v_{\text {max }}^{\prime}}\right]=\frac{2 E\left[L_{\text {road }}\right]}{E\left[v_{\text {max }}^{\prime}\right]} \\
& =\frac{2 E\left[L_{\text {road }}\right]}{E\left[v_{\max }\left(1-\lambda^{\prime} / \lambda_{\max }\right)\right]} \\
& =2 E\left[L_{\text {road }}\right] \times \frac{1}{v_{\max }} \times E\left[\frac{\lambda_{\max }}{\lambda_{\max }-\lambda}\right], \\
& =\frac{2 \lambda_{\max } E\left[L_{\text {road }}\right]}{v_{\max }} \times E\left[\frac{1}{\lambda_{\max }-\lambda_{s}}\right]
\end{aligned}
$$

where $L_{\text {road }}$ is the length of the road in meters. To determine $E\left[\frac{1}{\lambda_{\max }-\lambda_{s}}\right]$, consider $F_{\lambda_{s}}(x)=p\left(\lambda_{s} \leq x\right)=\frac{x}{\lambda_{\max }}$, which gives $f_{\lambda_{s}}(x)=\frac{1}{\lambda_{\max }}$. Then

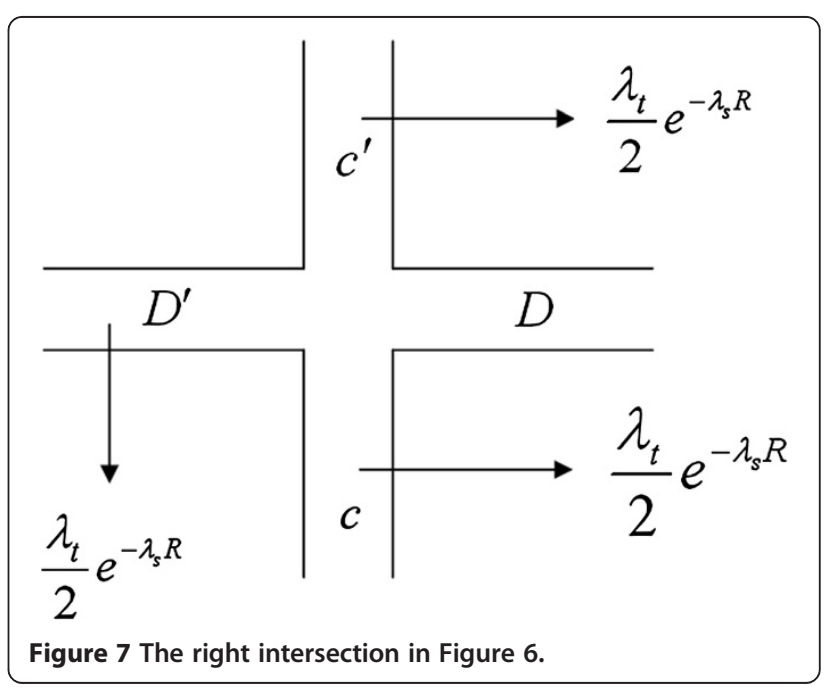




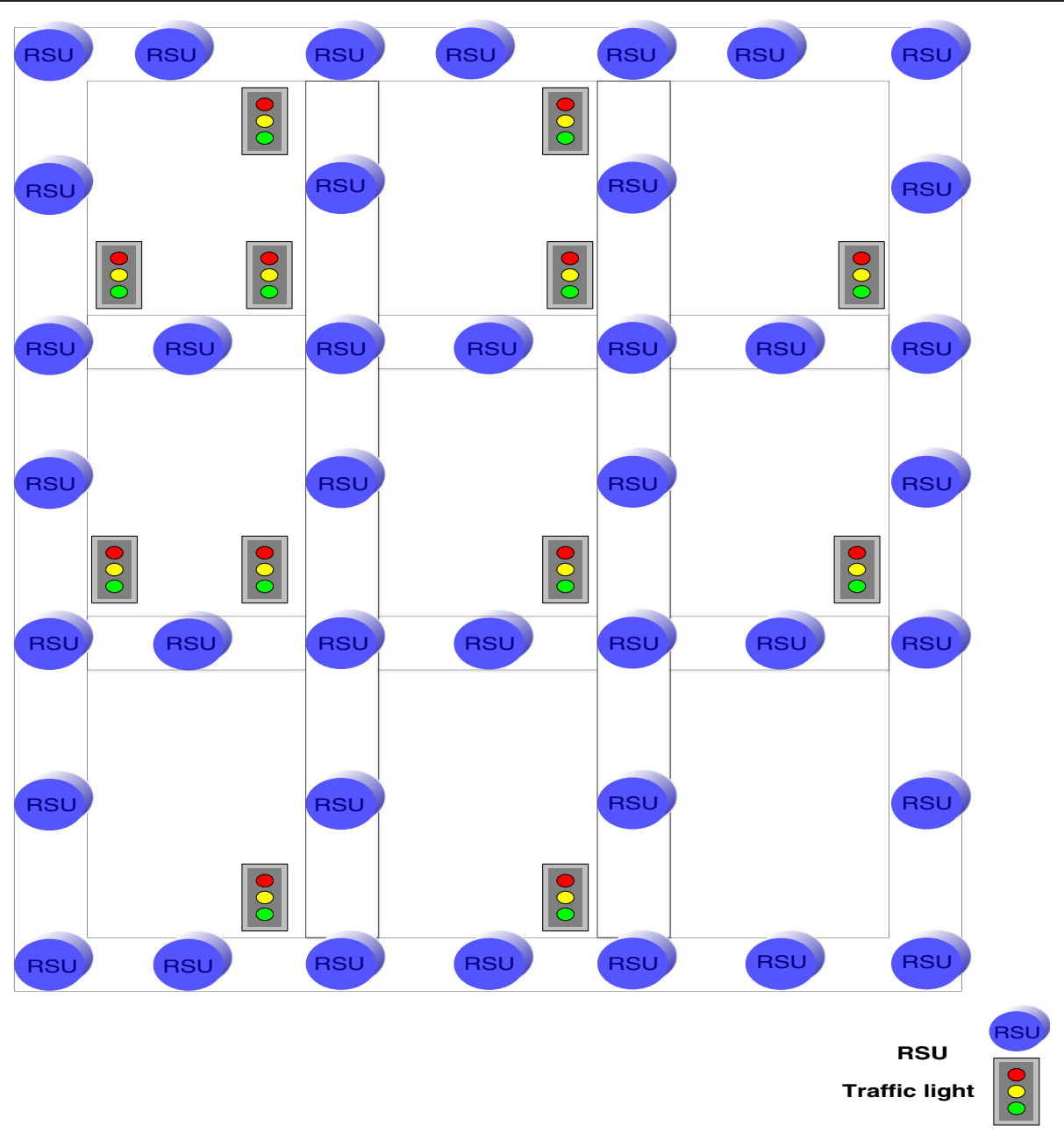

Figure 8 The grid topology with traffic lights at each intersection. The RSUs are located at every intersection and midway between intersections.

$$
\begin{aligned}
E\left[\frac{1}{\lambda_{\max }-\lambda_{s}}\right] & =\int_{0}^{\lambda_{a}} \frac{1}{\lambda_{\max }-\lambda_{s}} \times \frac{1}{\lambda_{\max }} d \lambda \\
& =\frac{1}{\lambda_{\max }} \ln \frac{\lambda_{\max }}{\lambda_{\max }-\lambda_{a}}
\end{aligned}
$$

We now consider $\lambda_{a}$, which is the minimum capacity such that the traffic remains clustered. Suppose a vehicle enters a congested road with capacity $\lambda_{\max }(\mathrm{veh} / \mathrm{m})$. In the case of maximum congestion, vehicle speeds are reduced to zero. With no movement towards the destination, the expected travel time increases significantly. Substituting $\lambda_{\max }$ in (12) results in infinity, which confirms this result. On the other hand, many vehicles are within communication range in a congested road, so packets can be propagated at maximum speed and with minimum delay.

Vehicles in a congested road can easily propagate data with minimum delay, resulting in long decoding distances.
Since the goal here is to investigate the effect of rateless coding on data dissemination, only partitioned traffic is considered. In this case, the distance between two successive clusters is greater than $R$. If this distance is $R$, the average cluster plus inter cluster length is

\section{Table 1 Simulation parameters}

\begin{tabular}{ll}
\hline Simulation time & $1000 \mathrm{~s}$ \\
Coverage area $(R)$ & $250 \mathrm{~m}$ \\
$\lambda$ per flow & $0.035 \mathrm{veh} / \mathrm{s}$ \\
Broadcast rate & 10 packets/s \\
Buffer size & 5000 packets \\
Number of RSUs & 40 \\
Number of messages that can be stored & 5 \\
Number of messages disseminated by an RSU & 4 \\
Number of packets to decode a message & 1000 \\
Number of vehicles & 960 \\
\hline
\end{tabular}




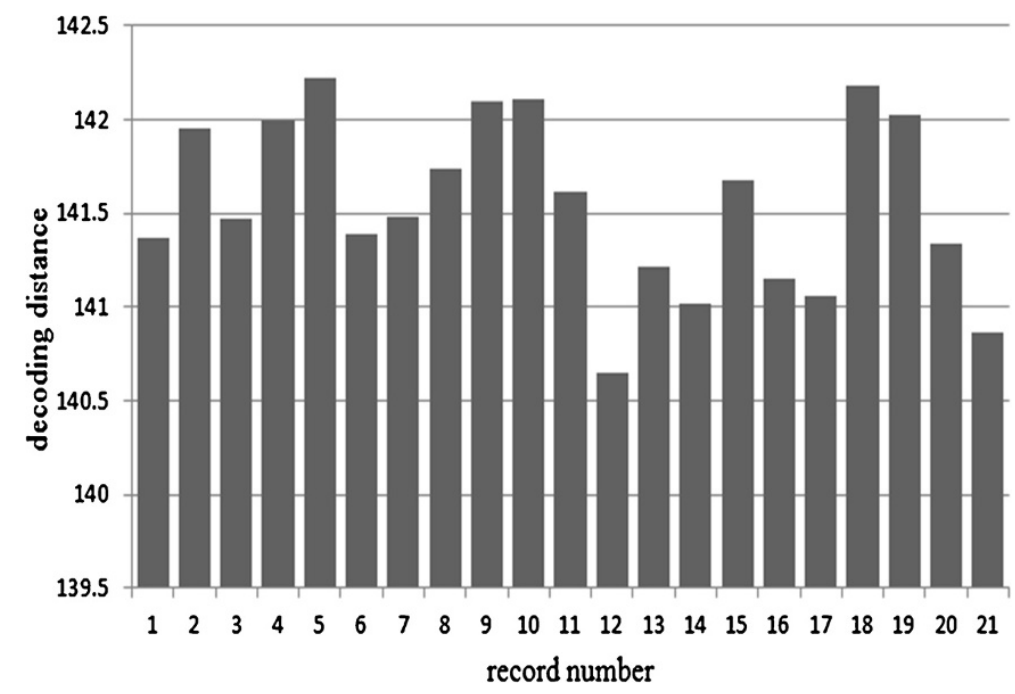

Figure 9 Decoding distances for messageB. The horizontal axis represents the $n$th vehicle (record number) that has decoded the message from the RSU.

$$
E\left[c_{L}\right]=R+\frac{1}{p_{d}}\left(\frac{1}{\lambda_{s}}-\frac{e^{-\lambda_{s} R}}{1-e^{-\lambda_{s} R}}\right)
$$

and if $\lambda_{s}=\lambda_{a}$, this becomes

$$
E\left[c_{L}\right]=R+\frac{1}{p_{d}}\left(\frac{1}{\lambda_{a}}-\frac{e^{-\lambda_{a} R}}{1-e^{-\lambda_{a} R}}\right) .
$$

The average number of clusters in a road in this case is

$$
\frac{E\left[L_{\mathrm{road}}\right]}{E\left[c_{L}\right]}=\frac{E\left[L_{\mathrm{road}}\right]}{R+\frac{1}{p_{d}}\left(\frac{1}{\lambda_{a}}-\frac{e^{-\lambda_{a} R}}{1-e^{-\lambda_{a} R}}\right)}
$$

and the corresponding average number of vehicles in a road is

$$
\frac{E\left[L_{\mathrm{road}}\right] \times E[c]}{R+\frac{1}{p_{d}}\left(\frac{1}{\lambda_{a}}-\frac{e^{-\lambda_{a} R}}{1-e^{-\lambda_{a} R}}\right)} .
$$

$\lambda_{a}$ can then be obtained by noting that

$$
\lambda_{a}=\frac{E[c]}{E\left[c_{\text {length }}\right]+E\left[s_{\text {intra }}\right]},
$$

which gives

$$
\lambda_{a} R p_{d}=\frac{\lambda_{a} e^{-\lambda_{a} R}}{1-e^{-\lambda_{a} R}},
$$

so that

$$
\lambda_{a} R p_{d}-e^{-\lambda_{a} R}=\lambda_{a} e^{-\lambda_{a} R}
$$

and consequently

$$
\lambda_{a}=\frac{1}{R} \ln \left(\frac{1+R p_{d}}{R p_{d}}\right) .
$$

Substituting $\lambda_{a}$ into the expression for $E\left[t_{L}\right]$ results in

$$
\begin{aligned}
E\left[t_{L}\right] & =\frac{2 \lambda_{\max } E\left[L_{\text {road }}\right]}{v_{\max }} \times \frac{1}{\lambda_{\max }} \times \ln \frac{\lambda_{\max }}{\lambda_{\max }-\lambda_{a}} \\
& =\frac{2}{v_{\max }} E\left[L_{\text {road }}\right] \times \ln \frac{\lambda_{\max }}{\lambda_{\max }-\frac{1}{R} \ln \left(\frac{1+R p_{d}}{R p_{d}}\right)} .
\end{aligned}
$$

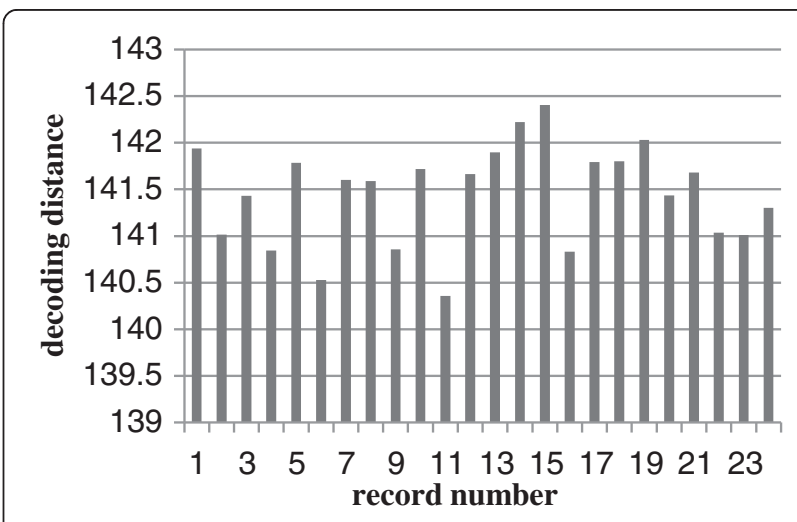

Figure 10 Decoding distances for messageB. The horizontal axis represents the $n$th vehicle (record number) that has decoded the message from the RSU. 


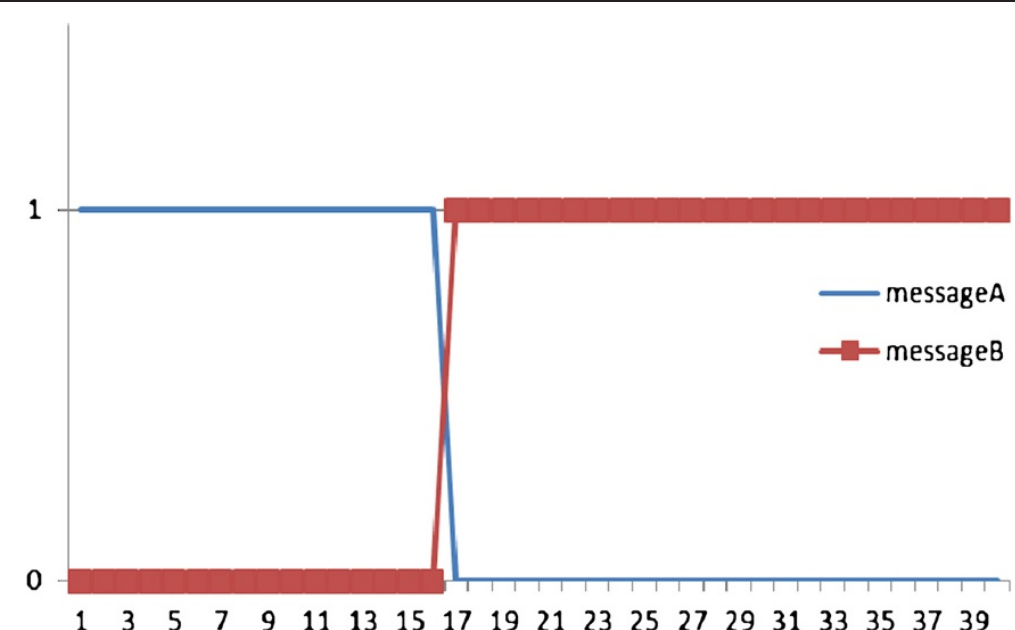

Figure 11 A switch in the message being disseminated for RSU961. The horizontal axis represents the $n$th vehicle that has decoded a message from the RSU.

\subsection{Intersection modeling}

Calculating the expected number of clusters encountered by a vehicle requires $E\left[N_{\text {clus inter }}\right]$, the expected number of clusters encountered by a vehicle in an intersection. This value is now determined. Consider the grid topology depicted in Figure 5, which has $m+1$ vertical roads and $n+1$ horizontal roads. To obtain an accurate network model, an intersection model is required. Figure 6 shows two intersections in the network. When a traffic light turns red, that road will no longer feed the intersection with vehicles, but it can be fed into because all roads are assumed to be bidirectional.

Assume that traffic lights $A$ and $A^{\prime}$ in the left intersection of Figure 6 are synchronized, and $B$ and $B^{\prime}$ are also synchronized. This is assumed for all similar pairs of intersection lights. Consider the left intersection. First suppose that traffic lights $A$ and $A^{\prime}$ are red. Then only roads $B$ and $B^{\prime}$ can feed $A^{\prime}$, so the vehicle arrival rate on $A^{\prime}$ from this intersection in this case is

$$
\begin{aligned}
\lambda_{A_{o}^{\prime}} & =\lambda_{B} \times p(\text { right turn })+\lambda_{B^{\prime}} \times p(\text { left turn }) \\
& =\frac{1}{3}\left(\lambda_{B}+\lambda_{B^{\prime}}\right),
\end{aligned}
$$

where $\lambda_{B}$ is the vehicle arrival rate on $B$ and $\lambda_{B}$ is the vehicle arrival rate on $B^{\prime}$, if the three possible directions are equiprobable.

Next, suppose that traffic lights $B$ and $B^{\prime}$ are red. In this case only road $A$ feeds $A^{\prime}$, so the vehicle arrival rate on $A^{\prime}$ from this intersection is

$$
\lambda_{A_{1}^{\prime}}=\frac{1}{3} \times \lambda_{A}
$$

The probability of a traffic light being red or green can be assumed to be 0.5 , therefore

$$
\begin{aligned}
E\left[\lambda_{A^{\prime}}\right] & =\left(\frac{1}{3}\left(\lambda_{B}+\lambda_{B^{\prime}}\right) \times \frac{1}{2}\right)+\left(\frac{1}{3} \times \lambda_{A} \times \frac{1}{2}\right) \\
& =\frac{1}{6}\left(\lambda_{A}+\lambda_{B}+\lambda_{B^{\prime}}\right) .
\end{aligned}
$$

Equation (22) is the arrival rate for road $A^{\prime}$. Assuming the arrival rate of vehicles on roads $A, B$ and $B^{\prime}$ is equal to $\lambda_{t}$, the corresponding cluster arrival rate is

$$
E\left[\lambda_{\text {clus }_{A^{\prime}}}\right]=\frac{\lambda_{t} / 2}{E\left[c_{\text {length }}\right]}=\frac{\lambda_{t}}{2} e^{-\lambda_{s} R} .
$$

Now consider the right intersection in Figure 6. Figure 7 shows this intersection with the corresponding cluster arrival rates. Consider a vehicle traveling along road $D$ toward this intersection. We then have

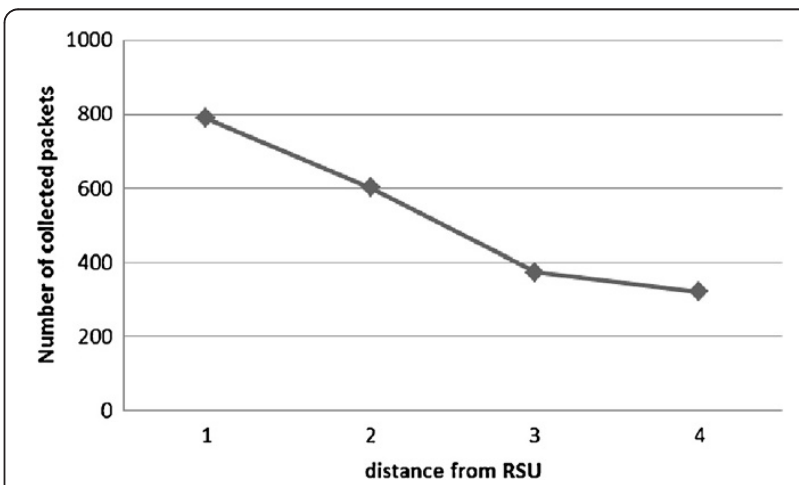

Figure 12 Data dissemination near RSU961 at a distance of 1 to $4 \mathrm{~km}$. 


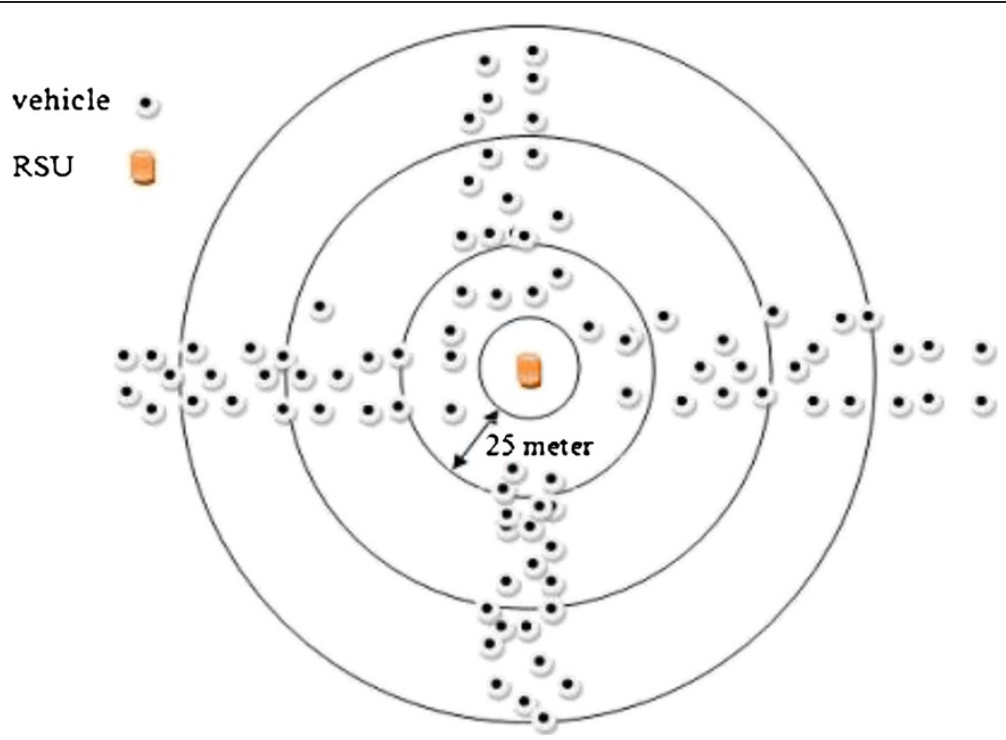

Figure 13 A typical intersection with vehicles traveling along the corresponding roads. The RSU is located at the center. The rings have radii a multiple of $25 \mathrm{~m}$.

$$
\begin{aligned}
E\left[N_{\text {clus inter }}\right]= & E\left[N_{\text {clus inter }} \mid C C^{\prime}=\operatorname{red}\right] p\left(C C^{\prime}=\mathrm{red}\right) \\
& +E\left[N_{\text {clus inter }} \mid A^{\prime} D=\operatorname{red}\right] p\left(A^{\prime} D=\mathrm{red}\right)
\end{aligned}
$$

If the lights at $C$ and $C^{\prime}$ are red, vehicles do not stop at the intersection. We now determine the number of clusters at the intersection. The vehicle arrival rates at $C$ and $C^{\prime}$ are $\frac{\lambda_{t}}{2} e^{-\lambda_{s} R}$, so the rate that vehicles arrive at the intersection is $2\left(\frac{\lambda_{t}}{2} e^{-\lambda_{s} R}\right)=\lambda_{t} e^{-\lambda_{s} R} \equiv \lambda_{t l}$, which is a Poisson process. The expected number of clusters waiting at an intersection is

$$
E\left[\text { clusters }_{\text {red }}\right]=\sum_{k=1}^{\infty} k \frac{e^{-\lambda_{t l}}\left(\lambda_{t l}\right)^{k}}{k !}=\lambda_{t l}
$$

Thus, if the traffic lights at $C$ and $C^{\prime}$ turn red, vehicles traveling along road $D$ toward the intersection will encounter $\lambda_{t l}$ clusters at this intersection.

When the traffic lights at roads $A^{\prime}$ and $D$ are red, vehicles will wait at the intersection for $T_{t l}$ seconds. Therefore,

$$
E\left[N_{\text {clus inter }} \mid A^{\prime} D=\mathrm{red}\right]=\frac{3}{2} \lambda_{t l} T_{t l},
$$

so that

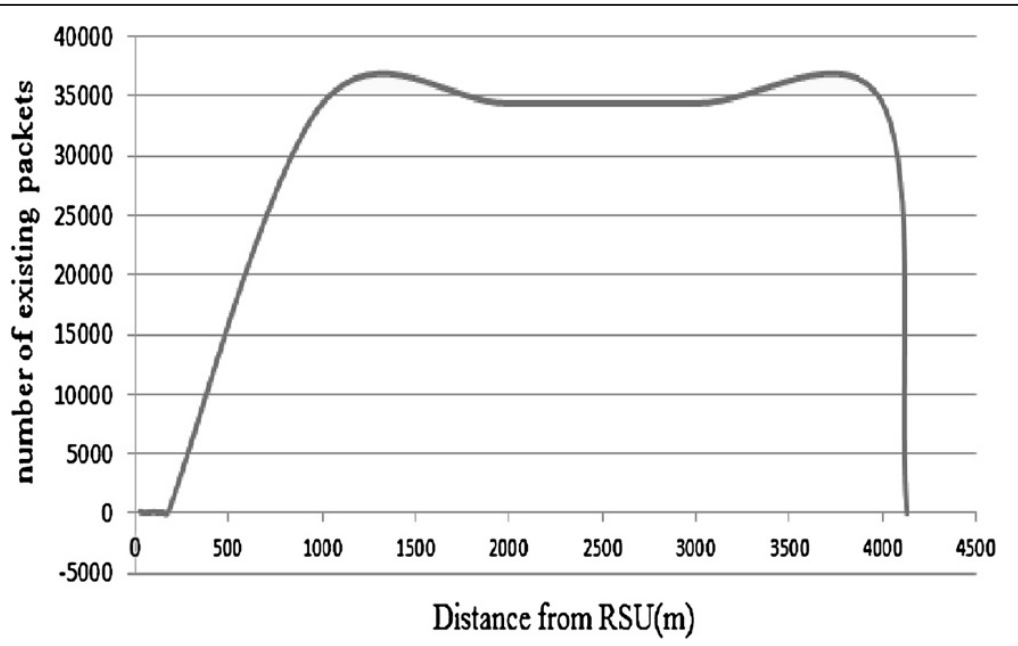

Figure 14 Data dissemination near an RSU midway between intersections. 


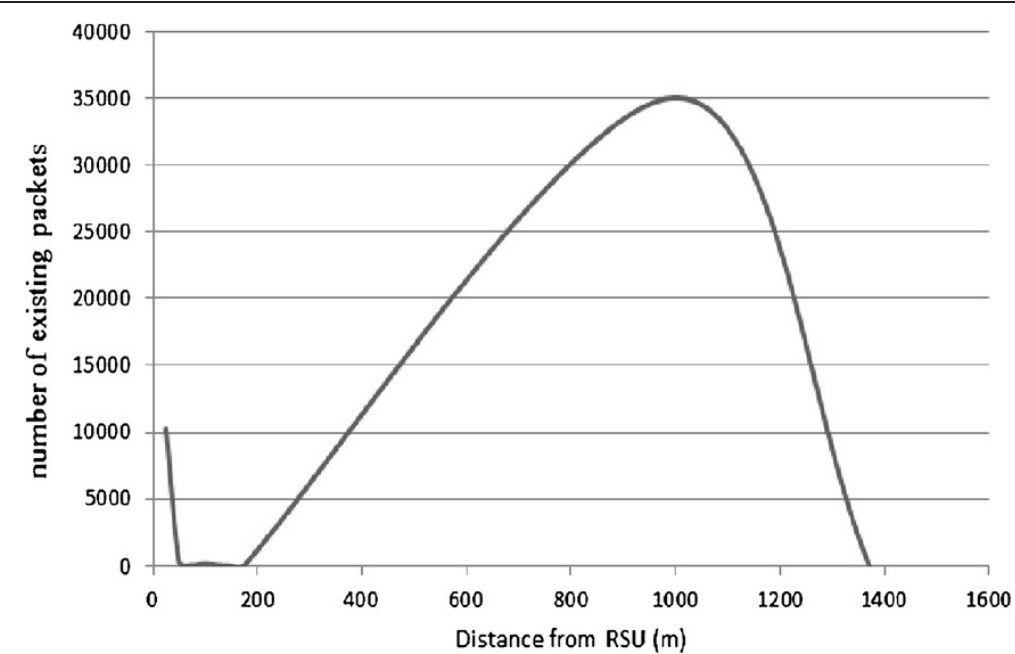

Figure 15 Data dissemination around an RSU at an intersection. The traffic light increases the number of carriers near the RSU.

$$
E\left[N_{\text {clus inter }}\right]=2 \lambda_{t l} T_{t l}
$$

Then from (10), we obtain

$$
\begin{aligned}
E\left[M_{n}(L)\right]= & \frac{L}{E\left[c_{\text {length }}\right]+E\left[s_{\text {inter }}\right]}+\lambda_{s} \frac{2 L}{v_{\max }^{\prime}}+E\left[N_{\text {clus inter }}\right] \\
= & \frac{L}{\left(1 / p_{d}-1\right)\left(1 / \lambda_{s}-\left(\operatorname{Re}^{-\lambda_{s} R} / 1-e^{-\lambda_{s} R}\right)\right)} \\
& +\lambda_{s} \frac{2 L}{v_{\max }^{\prime}}+2 \lambda_{t l} T_{t l}
\end{aligned}
$$

Suppose that each space in a vehicle buffer can hold $K$ packets, and a message can be decoded when $J$ distinct packets have been received $(J<K)$. The average number of roads and intersections that a vehicle must traverse to obtain a sufficient number of packets to decode a message is given by

$$
E\left[n_{\text {road }+ \text { inter }}\right]=\frac{J}{\frac{k}{2}\left(\left(\frac{E\left[L_{\text {road }}\right]}{\left(e^{\lambda_{s} R}-1\right)\left(\frac{1}{3}-\frac{R^{-\lambda}-\lambda_{s} R}{1-e^{-\lambda s} R}\right)+R+R+\frac{1}{\lambda}}+\lambda_{s} \frac{2 L}{v_{\max }^{\prime}}\right)+2 \lambda_{t l} T_{t l}\right)}
$$

The average time needed for a vehicle to collect $K$ different packets of a message is then obtained as

$$
\begin{aligned}
& E\left[T_{\text {total }}\right] \\
& =\frac{\frac{k}{2}\left(\left(\frac{E\left[L_{\mathrm{road}}\right]}{\left(e^{\lambda_{s} R}-1\right)\left(\frac{1}{3}-\frac{\operatorname{Re}^{-s_{s} R}}{1-e^{-\lambda_{s} R}}\right)+R+R+\frac{1}{\lambda}}+\lambda_{s} \frac{2 L}{v_{\max }^{\prime}}\right)+2 \lambda_{t l} T_{t l}\right)}{} \\
& \times \frac{2}{\nu_{\max }} E\left[L_{\text {road }}\right] \times \ln \left(\frac{\lambda_{\max }}{\lambda_{\max }-\frac{1}{R}}\right)
\end{aligned}
$$

We have that

$$
\lambda_{\text {clus }}=\frac{\lambda}{E\left[c_{\text {length }}\right]}=\frac{\lambda}{1 / p_{d}}=\lambda p d,
$$

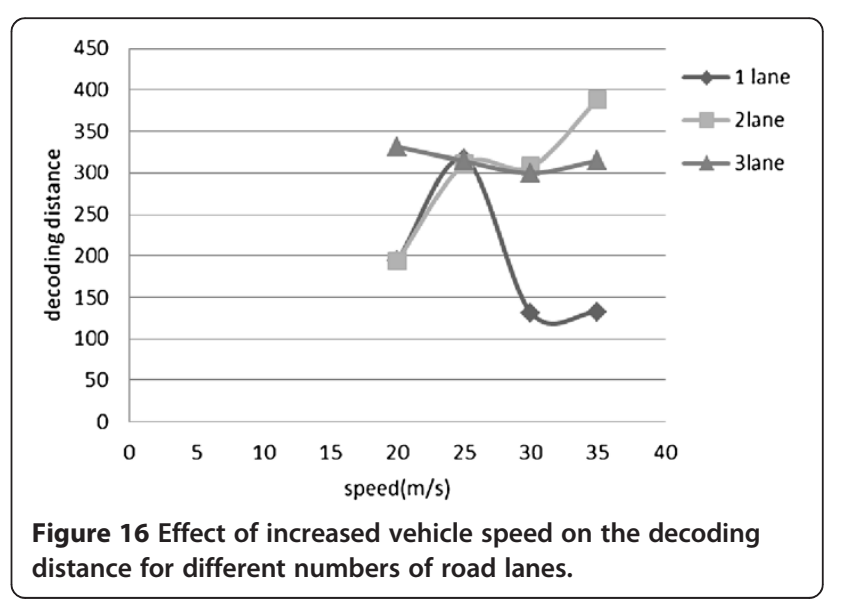




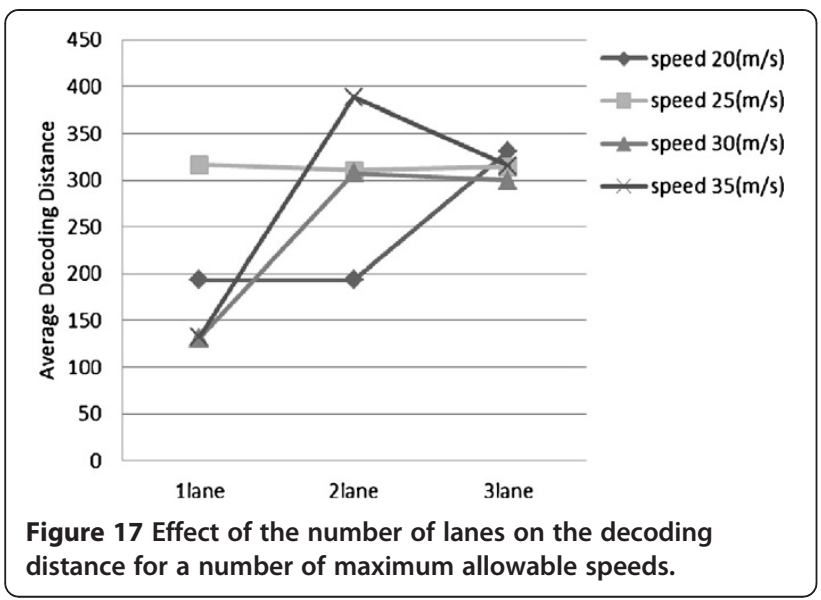

so the probability of successfully decoding a message is

$$
p_{\text {decode }}=\frac{e^{-\lambda p_{d} T_{\text {total }}}\left(\lambda p_{d} T_{\text {total }}\right) E\left[n_{\text {road }+ \text { inter }}\right]}{\left(E\left[n_{\text {road }+ \text { inter }}\right]\right) !} .
$$

\section{Simulation results and discussion}

In this section, we present simulation results to illustrate the performance of our system. The network topology employed is depicted in Figure 8. Vehicles enter the network from boundary intersections with inter-arrival times following an exponential distribution with parameter $\lambda$. The maximum speed on each road depends on the road traffic conditions. The value of $\lambda_{a}$ was chosen to avoid heavy traffic in the network. This is necessary so that vehicles travel in a clustered manner given the topology of the network.

There are 40 RSUs in the network located at the intersections and midway between intersections. All vertical road segments are 3-km long while the horizontal segments are 2-km long. Each RSU broadcasts four different messages during the simulation time with dissemination periods of $250 \mathrm{~s}$. Then the old messages are replaced with new ones. This replacement causes the vehicles to discard the packets related to the old messages and collect the new message packets instead.

The ns-2 network simulation package was used to evaluate the proposed scheme. Table 1 shows the simulation parameters. At the start of the simulation, 10\% of the cars are located at intersections in the network, and the remaining $90 \%$ can enter from the edges during the simulation time. The 960 vehicles are numbered 0 to 959 , and the RSUs are numbered 960 to 999 . RSU960 is located in the lower left corner of Figure 8, and RSU961 lies to its right.

First, the data dissemination from an RSU is examined. A limited vehicle buffer (space for five messages) with messages discarded as described previously is considered. The four transmitted messages are denoted as messageA, message $B$, message $C$, and message $\mathrm{D}$. We set $J=100$ so that the broadcast rate is 10 packets/s, and the message propagation rate is $1 / 10$ messages/s. The decoding distances of RSU961 for messageA and messageB are depicted in Figures 9 and 10, respectively. Figure 9 shows that 21 vehicles successfully decoded messageA of this RSU during the $1000 \mathrm{~s}$ simulation time, and Figure 10 shows that 24 vehicles decoded messageB. As stated previously, messageA remains valid for just the first 250 $\mathrm{s}$, and message $\mathrm{B}$ is disseminated for the next $250 \mathrm{~s}$. When a vehicle receives messageB packets from RSU961, the old packets from messageA are discarded and the new message packets are stored in their place. Figure 11 shows this transition according to the vehicles that have received messages from the RSU. In this figure, the horizontal axis denotes the $n$th vehicle that has decoded a message from the RSU. On the vertical axis, 1 denotes the message has been decoded, and 0 that it was not decoded.

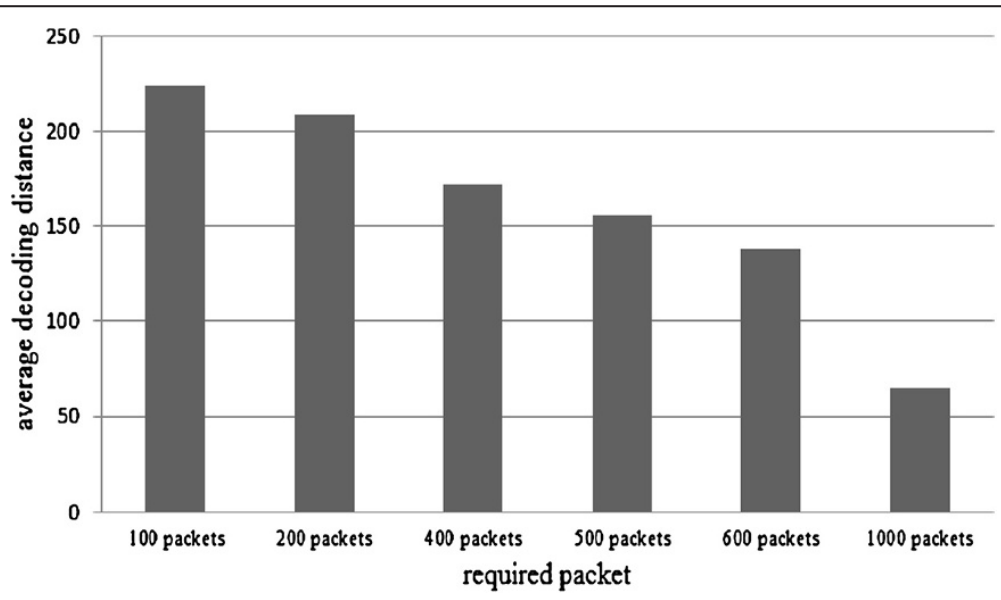

Figure 18 Effect of the number of packets required to decode a message on the average decoding distance. 
Table 2 Vehicle 14 buffer contents

\begin{tabular}{|c|c|c|c|c|c|c|c|c|c|}
\hline \multirow[t]{2}{*}{ Time } & \multicolumn{9}{|c|}{ RSU number } \\
\hline & 966 & 965 & 964 & 963 & 962 & 968 & 973 & 972 & 971 \\
\hline 100 & 1 & 1 & 1 & 0 & 0 & 0 & 0 & 0 & 0 \\
\hline 125 & 1 & 1 & 1 & 0 & 0 & 0 & 0 & 0 & 0 \\
\hline 150 & 1 & 1 & 1 & 1 & 0 & 0 & 0 & 0 & 0 \\
\hline 175 & 1 & 1 & 1 & 1 & 0 & 0 & 0 & 0 & 0 \\
\hline 200 & 1 & 1 & 1 & 1 & 0 & 0 & 0 & 0 & 0 \\
\hline 225 & 1 & 1 & 1 & 1 & 1 & 0 & 0 & 0 & 0 \\
\hline 250 & 0 & 1 & 1 & 1 & 1 & 1 & 0 & 0 & 0 \\
\hline 300 & 0 & 1 & 1 & 1 & 1 & 1 & 0 & 0 & 0 \\
\hline 325 & 0 & 1 & 1 & 1 & 1 & 1 & 0 & 0 & 0 \\
\hline 350 & 0 & 1 & 1 & 1 & 1 & 1 & 0 & 0 & 0 \\
\hline 375 & 0 & 0 & 1 & 1 & 1 & 1 & 1 & 0 & 0 \\
\hline 400 & 0 & 0 & 1 & 1 & 1 & 1 & 1 & 0 & 0 \\
\hline 425 & 0 & 0 & 1 & 1 & 1 & 1 & 1 & 0 & 0 \\
\hline 450 & 0 & 0 & 0 & 1 & 1 & 1 & 1 & 1 & 0 \\
\hline 475 & 0 & 0 & 0 & 1 & 1 & 1 & 1 & 1 & 0 \\
\hline 500 & 0 & 0 & 0 & 0 & 1 & 1 & 1 & 1 & 1 \\
\hline
\end{tabular}

The data disseminated pattern is investigated next. Suppose a vehicle is moving toward RSU961 and $J=1000$ packets are required to decode a message. Figure 12 shows that the number of packets in the vehicle buffer increases as the distance between the vehicle and RSU decreases. During the simulation time many vehicles decoded the messages of this RSU. These vehicles become carriers with respect to the RSU. This is illustrated in Figure 13 where rings with radii a multiple of $25 \mathrm{~m}$ are drawn around the RSU. These show the number of vehicles which have packets from the RSU as a function of distance. It is clear that more vehicles will become message carriers over time. Vehicles will eventually exit the $75 \mathrm{~m}$ ring, but will continue to carry message packets from this RSU.
However, these vehicles will eventually discard the message due to buffer limits or message replacement.

Figure 14 shows the pattern of data dissemination from an RSU located midway between intersections. In contrast, Figure 15 shows the corresponding pattern for an RSU located at an intersection. Typically, there are vehicles waiting at the intersection due to a red traffic light, so there are more vehicles near this RSU with packets from it.

\subsection{Effect of vehicle speed on decoding distance}

Recall that $t_{L}=2 L / v_{\max }^{\prime}$. When increases, vehicles traverse the road faster, so that $E\left[M_{n}(L)\right]$ decreases. Consequently, an increase in speed results in fewer encounters with vehicle clusters on a road. However, intersections must also be considered. For a vehicle waiting at a red light, as speeds increase, the number of vehicles that enter the intersection during this time interval will also increase. This results in more encounters with other vehicles. Thus, more packets will be collected at intersections. Therefore, an increased vehicle speed reduces the number of observed clusters on a road, but increases the number of observed clusters at intersections. Thus, the effects of changes in vehicle speed are complex. Figures 16 and 17 illustrate these effects for different numbers of lanes in each direction.

Figure 18 shows the average decoding distance with respect to the number of packets required to decode a message. As expected, this distance decreases as the number packets required for decoding increases.

The effect of discarding messages due to a limited buffer size is shown in Table 2. For example, at $250 \mathrm{~s}$ vehicle 14 discards a message from RSU966 because message packets are received from RSU961.

Finally, the impact of the data transmission rate is shown in Figure 19. The broadcast interval is the time between two successive transmissions. As expected, increasing this interval reduces the average decoding distance, but the effect is not substantial.

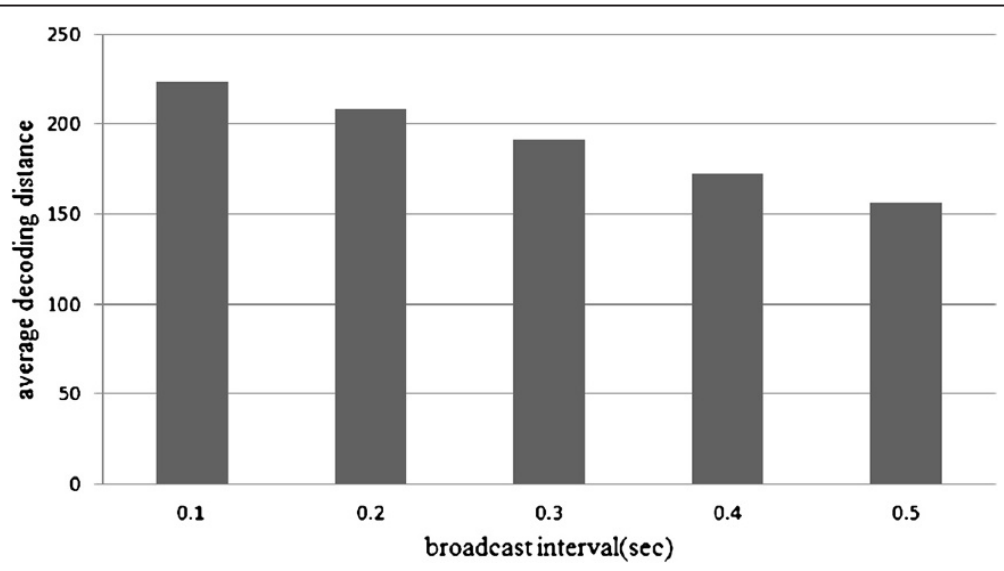

Figure 19 The effect of changing the time interval between two successive broadcasts on the decoding distance. Increasing the broadcast interval decreases the average decoding distance. 


\section{Conclusions}

In this article, rateless coding combined with the SCF data transfer technique was considered for VANETs in a grid topology. Vehicles were assumed to have limited buffer capacity, so old messages are discarded when the buffer is full. The decoding distance from an RSU was examined. An intersection model was proposed, and the effect of different parameters on the decoding distance was examined. Future research will consider improvements in the distribution of vehicles in the network and the effect of obstacles such as buildings on data propagation. The development of buffer management techniques to improve the probability of decoding should also be considered.

\section{Competing interests}

The authors declare that they have no competing interests.

\begin{abstract}
Authors' information
Mostafa Asgharpoor Salkuyeh received the M.Sc. degree from Isfahan University of Technology, Isfahan, Iran, in 2011. He is currently pursuing Ph.D. in Electrical and Computer Engineering at the Iran University of Science and Technology, Tehran, Iran. His research interests include vehicular ad hoc net-work (NANET), mobile ad hoc network (MANET) and cellular communication. Faramarz Hendessi received the M.Sc. degrees from Isfahan University of Technology, Isfahan, Iran and the Ph.D. degree from the Carleton University, Ottawa, in 1994. Since 1994, Dr. Hendessi has been with the faculty of the Electrical and Computer Engineering at the Isfahan University of Technology (http://www.iut.ac.ir) where he currently holds an Associate Professor position. He serves on the editorial board of the International Journal of Information and Communication Technology, Iran, and on the Technical Program Committees of several conferences. Prof. Hendessi's current research interests are in the areas of wireless communications and networking. Dr. Hendessi is Head of a well known IT Company in Isfahan, Iran (http://www.Payamnet.Com), which he established in 1995.

T. Aaron Gulliver received the Ph.D. degree in Electrical Engineering from the University of Victoria, Victoria, BC, Canada in 1989. From 1989 to 1991 he was employed as a Defence Scientist at Defence Research Establishment Ottawa, Ottawa, ON, Canada. He has held academic positions at Carleton University, Ottawa, and the University of Canterbury, Christchurch, New Zealand. He joined the University of Victoria in 1999 and is a Professor in the Department of Electrical and Computer Engineering. In 2002, he became a Fellow of the Engineering Institute of Canada, and in 2012 a Fellow of the Engineering Institute of Canada. His research interests include information theory and communication theory, algebraic coding theory, cryptography, and ultra wideband communications.
\end{abstract}

\section{Author details}

${ }^{1}$ Department of Electrical and Computer Engineering, Isfahan University of Technology, Isfahan 84156, Iran. ${ }^{2}$ Department of Electrical and Computer Engineering, University of Victoria, P.O. Box 3055, STN CSC, Victoria, BC V8W 3P6, Canada.

Received: 22 January 2012 Accepted: 1 March 2013

Published: 18 April 2013

\section{References}

1. JA Davis, AH Fagg, BN Levine, Wearable computers as packet transport mechanisms in highly-partitioned ad-hoc networks, in Proceedings of the International Symposium on Wearable Computing (Zurich, Switzerland, 2001), pp. $141-148$

2. P Deolasee, A Katkar, A Panchbudhe, K Ramamritham, P Shenov, Adaptive push-pull: disseminating dynamic web data, in Proceedings of the International Conference on World Wide Web (Hong Kong, 2001), pp. $265-274$
3. G Muhl, A Ulbrich, K Herrmann, T Weis, Disseminating information to mobile clients using publish-subscribe. IEEE Internet Comput. 8(3), 46-53 (2004)

4. M Johnson, L De Nardis, K Ramchandran, Collaborative content distribution for vehicular ad hoc networks, in Proceedings of the Allerton Conf. on Commun., Control and Computing (Monticello, IL, 2006), pp. 751-760

5. J-S Park, U Lee, SY Oh, M Gerla, DS Lun, Emergency related video streaming in VANET using network coding, in Proceedings of the ACM Workshop on Vehicular Ad hoc Networks (Los Angeles, CA, 2006), pp. 102-103

6. J Zhao, Y Zhang, G Cao, Data pouring and buffering on the road: a new data dissemination paradigm for vehicular ad hoc networks. IEEE Trans. Veh. Technol. 56(6), 3266-3277 (2007)

7. P Cataldi, A Tomatis, G Grilli, M Gerla, CORP: cooperative rateless code protocol for vehicular content dissemination, in Proceedings of the IFIP Mediterranean Ad Hoc Networking Workshop (Haifa, Israel, 2009), pp. 1-7

8. H Yu, H Ghajari, AN Playmoth, P Johansson, ViMesh (TM) high-speed V-band vehicular ad-hoc network, in Proceedings of the IEEE Military Communication Conference (Baltimore, MD, 2011), pp. 2123-2128

9. M Sardari, F Hendessi, F Fekri, DMRC: Dissemination of multimedia in vehicular networks using rate-less codes, in Proceedings of the IEEE INFOCOM Mobile Video Delivery Workshop (Rio de Janeiro, Brazil, 2009), pp. 1-6

10. M Sardari, F Hendessi, F Fekri, DDRC: data dissemination in vehicular networks using rate-less codes. J. Inf. Sci. Eng. 26(3), 867-881 (2010)

11. M Taheri, F Hendessi, Disseminating a large amount of data to vehicular network in an urban area. Int. J. Veh. Technol. 8(2010) (2011). Article ID 627968

12. KP Boyle, PJ Lin, CC Yu, Rateless unequal error protection codes for the additive Gaussian noise channel, in Proceedings of the IEEE Military Communication Conference (Boston, MA, 2009), pp. 1-6

13. M Luby, LT codes, in Proceedings of the IEEE Symposium on Foundations of Computer Science (Vancouver, BC, 2002), pp. 272-280

14. N Wisitpongphan, F Bai, P Mudalige, V Sadekar, O Tonguz, Routing in sparse vehicular ad hoc wireless networks. IEEE J. Sel. Areas Commun. 25(8), 1538-1556 (2007)

doi:10.1186/1687-1499-2013-106

Cite this article as: Salkuyeh et al.: Data dissemination with rateless coding in a grid vehicular topology. EURASIP Journal on Wireless Communications and Networking 2013 2013:106.

\section{Submit your manuscript to a SpringerOpen ${ }^{\odot}$ journal and benefit from:}

- Convenient online submission

- Rigorous peer review

- Immediate publication on acceptance

- Open access: articles freely available online

- High visibility within the field

- Retaining the copyright to your article

Submit your next manuscript at $>$ springeropen.com 